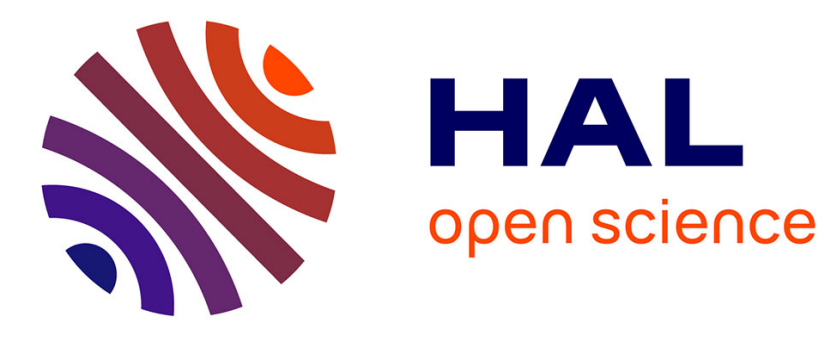

\title{
The quantum phase-transitions of water
}

François Fillaux

\section{To cite this version:}

François Fillaux. The quantum phase-transitions of water. EPL - Europhysics Letters, 2017, 119 (4), 10.1209/0295-5075/119/40008 . hal-01655365

\section{HAL Id: hal-01655365 https://hal.science/hal-01655365}

Submitted on 4 Dec 2017

HAL is a multi-disciplinary open access archive for the deposit and dissemination of scientific research documents, whether they are published or not. The documents may come from teaching and research institutions in France or abroad, or from public or private research centers.
L'archive ouverte pluridisciplinaire HAL, est destinée au dépôt et à la diffusion de documents scientifiques de niveau recherche, publiés ou non, émanant des établissements d'enseignement et de recherche français ou étrangers, des laboratoires publics ou privés. 


\title{
The quantum phase-transitions of water
}

\author{
FrançOis Fillaux ${ }^{1}$ \\ 1 Sorbonne Universités, UPMC Univ Paris 06, UMR 8233, MONARIS, F-7505 Paris, France
}

PACS 03.65.-w-Quantum mechanics

PACS 05.30.Rt - Quantum phase transitions

PACS 64.70.-p-Specific phase transitions

\begin{abstract}
It is shown that hexagonal ices and steam are macroscopically quantum condensates, with continuous spacetime-translation symmetry, whereas liquid water is a quantum fluid with broken time-translation symmetry. Fusion and vaporization are quantum phase-transitions. The heat capacities, the latent heats, the phase-transition temperatures, the critical temperature, the molar volume expansion of ice relative to water, as well as neutron scattering data and dielectric measurements are explained. The phase-transition mechanisms along with the key role of quantum interferences and that of Hartley-Shannon's entropy are enlightened. The notions of chemical bond and force-field are questioned.
\end{abstract}

Introduction. - Water, the matrix of life, covers the two-thirds of our planet. It is, therefore, of central importance across physics, chemistry, earth and life sciences, as well as to many technologies. Although water be likely the most extensively studied material, it is still a mystery. Many properties are said "anomalous", as they are different from those of other group $6 \mathrm{~A}\left(\mathrm{H}_{2} \mathrm{X}\right)$ and $4 \mathrm{~A}\left(\mathrm{H}_{4} \mathrm{X}\right)$ hydrides, or alcohols (ROH) [1. No other material is commonly found as solid (ice), liquid (water), or gas (steam). Steam is one of the lightest gas known, water is much denser than expected and ice is much lighter than expected. Water has the highest specific heat of all liquids, except ammonia, and its heat capacity is twice that of ice or steam. Proton tunnelling is observed in ice $\mathrm{I} h$ at $5 \mathrm{~K}$ [2] and in molecular jets of steam 3, 4], but not in water. Everyone is agreed that at least some of these "abnormal" properties stem from cooperative hydrogen bonds [5, but it is still not clear how this inter-linking translates to bulkscale behaviour and computer simulations are hardly conclusive, as they are notoriously sensitive to how the forces between molecules are modelled 6 .

Here, a radically new quantum framework is opposed to conventional wisdom, inspired by classical mechanics, that preexisting nuclear positions and energies can be measured in the three phases of water without perturbation, or through controllable modifications. Dimensionless nuclei are supposed to move along definite trajectories with definite positions and momenta, driven by definite forces, which are all regarded as elements of an objective reality at any time. Below, this deterministic paradigm is referred to as classical or local "microrealism". In this context, proton tunnelling in ice I $h$, or in gas-phase clusters, is regarded as interconversion of isoenergy configurations, along reaction-paths breaking and reforming hydrogen bonds 7,8 . However, this view is untenable, inasmuch as breaking and reforming bonds unavoidably destroys quantum coherence and tunnelling. In fact, nuclear quantum effects are outside the bounds of microrealistic determinism inherent to the Born-Oppenheimer separation of quantum electrons and classical nuclei. Tunnelling is relevant for nonnlocal, decoherence-free, nuclei exclusively. Tunnelling is, therefore, a direct evidence of the quantum nature of ice and steam. As a consequence of the lack of causal relationship between positions and momenta in quantum mechanics, tunnelling is not related to any definite nuclear trajectory usually referred to as "reaction path" or "mechanism".

Below, it is shown that "anomalous" properties are explained via pondering ice, water and steam as bulk-scale quantum systems. As a consequence, the physical phases of water shed light onto the long lasting question, dating back to Schrōdinger's cat, of the limits in size, temperature and complexity of the quantum theory applied to open systems [9].

The condensate. - The quantum framework for ice and steam is a follow up of previous works on various "hydrogen bonded" crystals 10 11. This framework rests on the notion of "condensate", that is a molar-scale superposition of every state that can be effectively, or in princi- 
ple, realized. This pure state has continuous spacetimetranslation symmetry. Nuclear positions and energy levels are indefinite. The wavefunction $\Phi \equiv 0$, the potential operator $\mathbb{V} \equiv 0$ and the statistical entropy $S \equiv 0$. For $\mathrm{H}_{2} \mathrm{O}$, the single-valued eigenenergy of the entangled degrees of freedom with respect to the ground state of the physical phase in question is $\mathcal{E}=\frac{9}{2} \mathcal{R} \Theta$. $\Theta$ is the temperature probed via projective measurements. It is different in nature, but not necessarily different in value, from the thermodynamic temperature of the surroundings, $T$. At thermal equilibrium, the condensate is stable, that is immune to decoherence, as long as $\mathcal{E}$ is lower than the Helmholtz energy of the statistical analogue [10]. There is no upper limit in temperature, insofar as the continuous spacetime translation symmetry is preserved.

At the microscopic level, an incoming wave breaking the continuous translation-symmetry realizes a transitory state that is not an eigenstate of the condensate. The condensate as a whole is virtually unaffected by a single event and spontaneous decay of the induced instability destroys quantum correlations with the outgoing wave. The condensate is, therefore, immune to decoherence induced by a measurement, or by the environment. As long as the probability for an incoming wave to entangle with a state previously realized is insignificant, the outcome of a measurement is independent of previous events. Every single realization can be projected onto the eigenstates of a context-dependent operator. On the one hand, the outcome of elastic scattering events breaking the continuous space-translation symmetry yields the static probability densities in the " $x$-space" of the nuclear coordinates, while the time variable and the vibrational states remain indefinite. On the other hand, energy transfer breaking the time-translation symmetry realizes eigenstates of the potential operator in the $\chi$-space of the eigenvectors of the Hamiltonian operator, while nuclear positions are indefinite. The potential operator in $\chi$-space is nonlocal. It is different in nature from the Born-Oppenheimer potential in $x$-space. Because elastic or inelastic events are unpredictable and mutually exclusive, there is no causal relationship between diffraction and spectroscopy (Bohr's complementarity principle) 12. Nuclear trajectories are ruled out.

Quantum versus classical. - The heat capacity $\frac{9}{2} \mathcal{R}$ of a $\mathrm{H}_{2} \mathrm{O}$ condensate is half that of a statistical network of tetra-coordinated molecules, that is $9 \mathcal{R}$ in the (high temperature) regime of equipartition. It turns out that $C_{p I h}\left(T_{F}\right) \approx 37.77 \mathrm{~J} \cdot \mathrm{mol}^{-1} \cdot \mathrm{K}^{-1}$ measured for ice $\mathrm{I} h$ at the melting point $T_{F}=273.16 \mathrm{~K}$ at standard pressure $\left(P_{a}\right)$ [13, is $\approx 1.01 \times \frac{9}{2} \mathcal{R}\left(\mathcal{R}=8.314 \mathrm{~J} \cdot \mathrm{mol}^{-1} \cdot \mathrm{K}^{-1}\right)$. Ice $\mathrm{I} h$ can be regarded as a quasi-pure condensate.

Quantum condensation is of consequence to the structure of ice $\mathrm{I} h$, as commonly represented in textbooks. This structure consists of an ordered lattice of buckled hexagons with oxygens at the corners, while the protons at a single site off-center each $\mathrm{O} \cdots \mathrm{O}$ bond lack any kind of long-range order. Bernal and Fowler 14 argued that water molecules obey the "ice rules" and Pauling 15 evaluated the residual entropy $S_{\mathrm{I} h}=\mathcal{R} \ln \frac{3}{2}$ that persists down to the lowest temperature measured 16]. However, this residual entropy is in defiance of the third law of thermodynamics. On the contrary, the zero-entropy of the condensate at thermal equilibrium complies with the third law without conflict with the ice rules, since nuclear positions are indefinite. The double peak of the proton density revealed by neutron diffraction [17] is not due to statistical disordering. It witnesses to the non-separability of proton sites which are equally occupied by "half-protons" in terms of probability densities. Regarding calorimetric measurements, in the aftermath of a $T$-jump, heat transfer yields a transitory mixture of states, until complete relaxation to the condensed state at $T+\Delta T$. The residual entropy and hence the ice rules are contextual: they are relevant out of thermal equilibrium only.

The heat capacity of liquid $\mathrm{H}_{2} \mathrm{O}$ in the range $T_{F}-T_{B}$ $\left(T_{B}=373.16 \mathrm{~K}\right.$ is the boiling temperature at $\left.P_{a}\right)$ is nearly a constant, with a shallow minimum at $T_{m C h} \approx 36^{\circ} \mathrm{C}$. The mean value $C_{p w h}=75.65 \pm 0.36 \mathrm{~J} \cdot \mathrm{mol}^{-1} \cdot \mathrm{K}^{-1} 18$, namely $(1.010 \pm 0.005) \times 9 \mathcal{R}$, is consistent with tetrahedral coordination, virtually temperature independent. This is apparently at variance with Bol's conclusion about water at $25^{\circ} \mathrm{C}$, based on x-ray diffraction: "each molecule is not surrounded by 4 hydrogen-bonded neighbours but by a somewhat smaller number, probably 3.2 (on average)..." 19. Similarly, Wernet et al. 20 deduced from x-ray absorption spectroscopy that molecules in water bind on average to $2.2 \pm 1.0$ others. However, these interpretations are misleading since the electronic-correlation-degree $\alpha_{w}$ determined via $\mathrm{x}$-ray is, in actual fact, different in nature from the coordination degree derived from $C_{p w h}$.

The minimum heat capacity of liquid $\mathrm{D}_{2} \mathrm{O}$ is $C_{p w d} \approx$ $82.58 \mathrm{~J} \cdot \mathrm{mol}^{-1} \cdot \mathrm{K}^{-1} \approx 1.10 \times 9 \mathcal{R}$ at $T_{m C d} \approx 61^{\circ} \mathrm{C} 21$. This value reveals correlations outside the bounds of the equipartition theorem (see below).

The heat capacity of steam at $T_{B}$ is that of a condensate: $C_{p v} \approx 37.47 \mathrm{~J} \cdot \mathrm{mol}^{-1} \cdot \mathrm{K}^{-1} 22$, i.e. $\approx 1.001 \times \frac{9}{2} \mathcal{R}$. This value is outside the bounds of the kinetic theory of gases, namely $6 \mathcal{R}$ for free $\mathrm{H}_{2} \mathrm{O}$ and $9 \mathcal{R}$ for tetra-coordinated $\mathrm{H}_{2} \mathrm{O}$. Tiny temperature effects on $C_{p v}$ suggest that the condensate survives up to at least the critical temperature $T_{c} \approx 647.1 \mathrm{~K}$.

The condensate framework is in defiance of the common wisdom that phases sharing boundaries with liquid water have disordered hydrogen bonding. In addition, it questions attempts to learn about the liquid from the study of (small) gas-phase clusters [4].

Fusion. - In addition to evidences based on heat capacity data, the condensate-to-statistical character of fusion is corroborated by the mean kinetic-energy of protons, say $\bar{K}(T)$, measured via neutron Compton scattering (NCS) 23. In ice, $\bar{K}_{\mathrm{I} h}(T)$ is practically a constant between $5 \mathrm{~K}$ and $271 \mathrm{~K}$, as the induced zero-point energy 
is not thermalized on the NCS timescale $\left(\sim 10^{-15} s\right)$. In water, the temperature law $\bar{K}_{w}(T) \approx K_{w 0}+0.1 \times T$, in meV units, accords with that for preexisting oscillators at thermal equilibrium: $\bar{K}_{w}(T)=K_{w 0}+\frac{3}{2} \mathcal{R} T\left(\frac{3}{2} \mathcal{R} \approx 0.12\right.$ $\left.\mathrm{meV} \cdot \mathrm{mol}^{-1} \cdot \mathrm{K}^{-1}\right)$

The protons of $\mathrm{H}_{2} \mathrm{O}$ molecules in water are distinguishable. The equilibrium entropy is $S_{w}=2 \mathcal{R} \ln \frac{3}{2} \approx 6.74$ $\mathrm{J} . \mathrm{mol}^{-1} \cdot \mathrm{K}^{-1}\left(\approx 6.6177 \mathrm{~J} \cdot \mathrm{mol}^{-1} \cdot \mathrm{K}^{-1} \approx 0.98 \times S_{w}\right.$ measured at $298 \mathrm{~K})$ and the enthalpy $T_{F}\left(S_{w}-S_{\mathrm{I} h}\right) \approx 921$ J.mol ${ }^{-1}$ is marginal relative to the heat of fusion $\Delta H_{F} \approx$ $6007 \mathrm{~J}^{-\mathrm{mol}^{-1}}$ measured at $P_{a} 13$.

Consider a single-step transition from the condensate - eigenenergy $\mathcal{E}_{1 h}\left(T_{F}\right)=\frac{9}{2} \mathcal{R} T_{F}$, entropy $S_{1 h}=0$ - to the liquid - Helmholtz energy $A_{w}\left(T_{F}\right)=T_{F} \mathcal{R}\left[9-2 \ln \frac{3}{2}\right]$. The enthalpy $\Delta H=\mathcal{R} T_{F}\left(\frac{9}{2}+2 \ln \frac{3}{2}\right) \approx 12061 \mathrm{~J}^{\mathrm{mol}}{ }^{-1}$, is $\approx 2.01 \times \Delta H_{F}$. In fact, because $\mathcal{E}_{\mathrm{I} h}\left(T_{F}\right) \neq 9 \mathcal{R} T_{F}$, the two phases cannot be in contact at thermal equilibrium, so a stepwise process through an intermediate state is necessary to get over the energy difference. This can be thought of as: step (A) endothermic transfer of $\Delta H / 2 ;$ step (B) internal conversion of $-\Delta H / 2$.
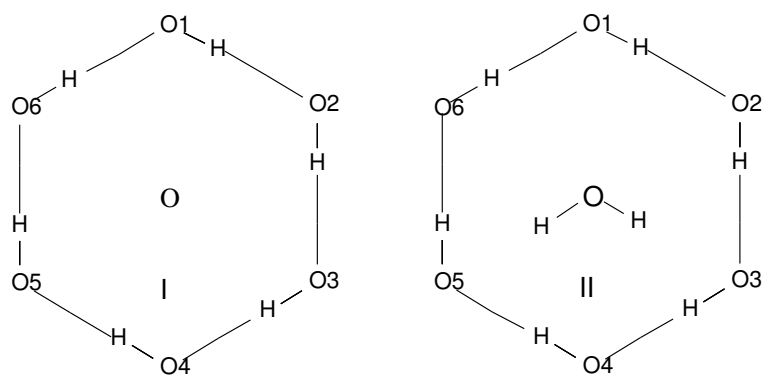

Fig. 1: Geometric graphs for a water molecule inside a clockwise hexamer. Lines are guides for the eyes. Only intracage vertexes are shown.

The entropy of the intermediate step corresponds to $\exp \left[\frac{9}{4}+\ln \frac{3}{2}\right] \approx 14.23$ distinguishable bulk-scale states realized via disentanglement of the ice condensate. In principle, there is no graphical representation of the molecular structures for such nonlocal states. However, the symmetry operators of the 14 states accounting for $\approx 98 \%$ of the entropy are isomorphic to the symmetry group of the topological graphs comprised of an interstitial vertex arbitrarily labeled "O" at the centers of clockwise and anticlockwise hexagons with distinguishable vertexes labeled "O-H" (fig. 11). Graph I has six-fold symmetry, so it is single. Graph II is one among six equivalent graphs necessary to comply with the six-fold symmetry. The upper bound of $\approx 2 \%$ for statistical deviations practically $\mathrm{ex}-$ cludes further topologies.

The reversed step $\mathbf{A}$ of crystallization is condensation of the 14 states. The ice condensate can be represented by $\left|\Phi_{\mathrm{I} h}\right\rangle=\sum_{i=1}^{14}\left|\Psi_{i}\right\rangle$, with $\left|\Psi_{i}\right\rangle=28^{-1 / 2}\left\{|\operatorname{Hex}\rangle+\left|\operatorname{Int}_{i}\right\rangle\right\}$, $\langle\operatorname{Hex} \mid \operatorname{Hex}\rangle=\mathcal{N}_{\mathrm{A}} / 14,\left\langle\operatorname{Int}_{i} \mid \operatorname{Int}_{j}\right\rangle=\delta_{i j} \mathcal{N}_{\mathrm{A}} / 14,\left\langle\operatorname{Hex} \mid \operatorname{Int}_{i}\right\rangle=$ $\left\langle\operatorname{Int}_{i} \mid \mathrm{Hex}\right\rangle=0 . \mathcal{N}_{\mathrm{A}}$ is Avogadro's constant. The probabilities to realize a state isomorphic with III or IV in
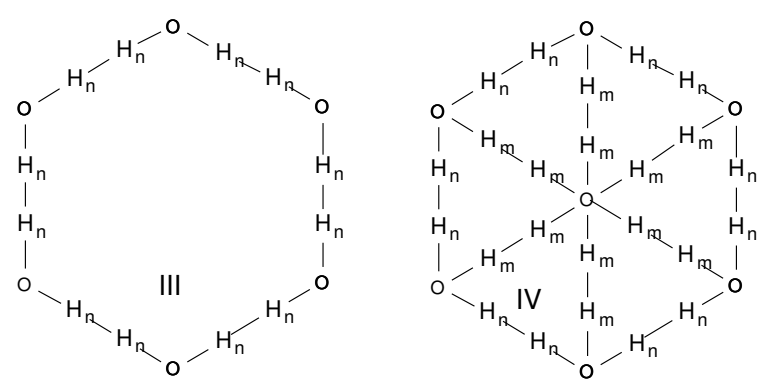

Fig. 2: Geometric graphs of ice $\mathrm{I} h$. Lines are guides for the eyes. Only intracage vertexes are labeled. Site occupancies are 1 for $\mathrm{O}, n=1 / 2, m=1 / 12$ for $\mathrm{H}$.

fig. 2 are $\rho_{\mathrm{Hex}}=\mathcal{N}_{\mathrm{A}}^{-1} \sum_{i=1}^{14} \sum_{j \neq i}^{14}\left\langle\Psi_{i} \mid \Psi_{j}\right\rangle=13 / 14$ or $\rho_{\text {Int }}=\mathcal{N}_{\mathrm{A}}^{-1} \sum_{i=1}^{14}\left\langle\Psi_{i} \mid \Psi_{i}\right\rangle=1 / 14$, respectively. Quantum interferences account for the lack of visibility of the intercalated lattice.

Step B of fusion merges the 14 states into a disentangled ensemble of nonlocal oxygen atoms and two distinguishable nonlocal protons per oxygen, isomorphic with IV in fig. 2. Water is a bulk-scale molecule of molar stoichiometry $\left[\left(\mathrm{H}_{2} \mathrm{O}\right)_{7}\right]_{\mathcal{N}_{\mathrm{A}} / 7}$, for which the usual notions of individual atoms, or ions, or molecules, or clusters, subject to many-body deterministic correlations via definite forces are ruled out. Hydrogen bonds, ice rules and tetrahedral coordination do not exist. Positional disorder is meaningless.

In the $\chi$-representation of water, the so called "protonstretching-modes" (coordinates $\chi_{j}, j=1 \cdots 7$ ) are eigenstates of asymmetric double-wells, which can be treated as two-level systems under normal $P, T$-conditions. The asymmetry is the energy difference $h \nu_{1}$ between the lower states localized in either $L$ or $R$ well, say $\left|0_{L}\right\rangle$ and $\left|1_{R}\right\rangle$ for $E_{R}-E_{L}=h \nu_{1}$, or $\left|0_{R}\right\rangle$ and $\left|1_{L}\right\rangle$ for $E_{L}-E_{R}=h \nu_{1}$ (see fig. 5 in 10]) The 14 asymmetric double-wells of the intermediate state merge into 7 pairs of disentangled double-wells with opposite asymmetry for every $\chi_{j}$. In ice, entanglement yields 7 tunnelling splittings due to tiny delocalization of the wavefunctions: $\left|0_{ \pm j}\right\rangle=2^{-1 / 2}\left[\left|0_{L J}\right\rangle \pm\left|0_{R j}\right\rangle\right]$; $\left|1_{ \pm j}\right\rangle=2^{-1 / 2}\left[\left|1_{L j}\right\rangle \pm\left|1_{R_{j}}\right\rangle\right] ; h \nu_{0 \pm j}=h \nu_{1 \pm j}=h \nu_{t} \forall j$.

Fusion occurs when $9 k_{B} T_{F}=7 h\left(\nu_{1}-\nu_{t} / 2\right) . k_{B}$ is Boltzmann's constant. At that temperature, energy transfer to the states $\left|0_{-j}\right\rangle$ of ice yields, via the intermediate states graphed in fig. 11, the disentangled states $\left|1_{L j}\right\rangle$ and $\left|1_{R j}\right\rangle$ of water. Then, the limited residence time in either well breaks the continuous time-translation symmetry. In classical mechanics, because positions and energy are causally related, fluctuations, e.g. phonons, do not necessarily destroy the spacetime coherence of a crystal lattice. In quantum mechanics, by contrast, noncausal fluctuations unavoidably yield a quantum fluid at $T_{F}$. Water is a mixture of states $\sum_{\chi, n} p_{\chi, n}\left|\phi_{\chi, n}\right\rangle\left\langle\phi_{\chi, n}\right|$ with continuous space-translation symmetry in $x$-space for each eigenstate $n$ of each degree of freedom $\chi$ and $p_{\chi, n}$ is Boltzmann's 
population factor.

For $\mathrm{D}_{2} \mathrm{O}, h \nu_{t d} \approx 0 . T_{F d} \approx 276.98 \mathrm{~K}$ and $T_{F d}-T_{F h} \approx$ $3.82 \mathrm{~K}$, gives $h \nu_{t F} \approx 1.09 \mathrm{~K}$. This value is consistent with QENS measurements [2] (see below). The isotope effect accords with tunnelling in $7 \times 1-\mathrm{D}_{\chi}$ distinctive of IV (fig. 2 in ice $\mathrm{I} h$ and with the same 7 -fold dimensionality in water, but without tunnelling. On the other hand, $h \nu_{1} \approx 29$ meV compares favourably with a maximum of the densityof-states reported at $\approx 28 \mathrm{meV}$ in the inelastic neutronscattering spectrum of ice 24 and with $h \nu_{1} \approx 27 \mathrm{meV}$ for potassium hydrogen carbonate $\left(\mathrm{KHCO}_{3}\right) 25$ or $h \nu_{1} \approx 28$ meV for potassium dihydrogen phosphate $\left(\mathrm{KH}_{2} \mathrm{PO}_{4}\right)$ [10.

It turns out that $T_{F h}, T_{F d}$ and $\Delta H_{F}$ are totally determined by $h \nu_{1}, h \nu_{t F}$ and the dimensionality of the "protonstretching-modes".

Ice. - The state vector $\left|\Phi_{\mathrm{I} h}\right\rangle$ explains several properties.

(i) $\rho_{\text {Int }} \approx 0.07$ accords qualitatively with the low visibility of interstitial oxygen in the partial structure factor $S_{\mathrm{OO}}(\mathbf{Q})$ of ice I $h$ 26.

(ii) A QENS event in the temperature range $h \nu_{t} \ll$ $k_{B} T \ll h \nu_{1}$ realizes with probability $\rho_{\text {Int }}$ a transient superposition $\sum_{j=1}^{7} 2^{-1 / 2}\left[\left|0+{ }_{j}\right\rangle+\left|0-{ }_{j}\right\rangle\right][10$. The probability $\rho_{\text {Int }} / 2 \approx 0.036$ matches the reported QENS intensity ratio of $\approx 4 \%$ relative to the elastic peak. There is no evidence to suggest tunnelling in $6 \times 1-\mathrm{D}_{\chi}$, isomorphic with III, on the QENS-timescale. The measured relaxation rate at $5 \mathrm{~K}\left(k_{t} \approx 2.7 \times 10^{11} \mathrm{~Hz}\right)$ yields the tunnelling frequency $\nu_{t h}=k_{t} / 14 \approx 1.93 \times 10^{10} \mathrm{~Hz}$ for each of the $7 \chi_{j}$ 's. $h \nu_{t h} / k_{B} \approx 0.97 \mathrm{~K}$ is quite similar to $h \nu_{t F}$ and it is undecidable whether or not $h \nu_{t F}>h \nu_{t h}$ is significant, as statistical errors on $k_{t}$ are unknown. Whatever the case, tunnelling splittings survive up to $T_{F h}$, in defiance of the semiclassical phonon-assisted tunnelling scheme. $h \nu_{t h} / k_{B}$ accords with the very weak temperature effect on the QENS intensity. By contrast, concerted transfer of six protons represented by a collective coordinate in 1-D advocated by Bove et al. 2 should yield a greater splitting, $h k_{t} /\left(2 k_{B}\right) \approx 7 \mathrm{~K}$, for a greater effective mass $\geq 6$ amu and an exceedingly low potential barrier incompatible with stretching frequencies $>400 \mathrm{meV}$. In addition, the QENS intensity for concerted tunnelling should be divided by a factor of $\approx 4$ between $100 \mathrm{~K}$ and $5 \mathrm{~K}$, in contrast with observations. Besides, even from the microrealistic viewpoint, collective tunnelling around the rings is sterically excluded by the inter-distances of proton-sites greater than $1 \AA$ and the oxygen atoms at the corners. Path integral simulations in $x$-space were claimed to confirm the concerted transfer of six protons 8 but, in actual fact, tunnelling distinctive of the $\chi$-space is simply out of reach of such simulations.

A quantitative argument in favour of concerted tunneling put forward by Bove et al. is the lack of QENS signal for a deuteration degree $\rho=0.20$. However, this observation is also consistent with IV in fig. 2, since tunnelling should vanish as a single proton among 7 is replaced by a deuteron, so the probability for tunnelling is $(1-\rho)^{7} \approx 0.2$. In addition, the QENS width proportional to $(1-\rho)$ further diminishes the visibility of the signal 10 .

(iii) The quasi-linear decrease of $C_{p I h}(T)$ from $T_{F}$ to the lowest temperature measured $(\approx 20 \mathrm{~K}) 27$ is in defiance of microrealistic models. In the quantum theory, $C_{p I h}(T) \approx C_{p I h}\left(T_{F}\right) T / T_{F}, T \leq T_{F}$, means that a fraction $T / T_{F}$ of the condensate is at $\mathcal{E}_{F}$, while the fraction $\left(1-T / T_{F}\right)$ is frozen out at $\mathcal{E}=0$. There are only two stationary states because thermalization (via elastic events) is permitted at $\mathcal{E}_{F}$ exclusively. The heat transferred to the crystal via disentanglement is $d H=9 \mathcal{R}\left(T / T_{F}\right) d T$ and $\int_{0}^{T} d H=\frac{9}{2} \mathcal{R} T^{2} / T_{F}=\mathcal{E}(T)$.

(iv) The transition $\mathrm{I} h \longleftrightarrow \mathrm{XI}$, say at $T_{0}$, can be sketched as follows. Cooling ice $\mathrm{I} h$ across $T_{0}$ split the symmetric double-wells into the asymmetric double-wells of the orthorhombic (dielectric) ice XI, in which only every other site is occupied by a proton or a deuteron. Dielectric measurements of ice XI domains in quenched $\mathrm{I} h$ XI mixtures 28 read: $T_{0 h}(\mathrm{I} h \longrightarrow \mathrm{XI})=58.9 \mathrm{~K}$ (cooling) and $T_{0 h}^{\prime}\left(\mathrm{XI} \longrightarrow h_{r}\right)=73.4 \mathrm{~K}$ (heating) for $\mathrm{H}_{2} \mathrm{O}$; $T_{0 d}(\mathrm{I} h \longrightarrow \mathrm{XI})=63.7 \mathrm{~K}$ (cooling) and $T_{0 d}^{\prime}\left(\mathrm{XI} \longrightarrow h_{r}\right)=$ $78.2 \mathrm{~K}$ (heating) for $\mathrm{D}_{2} \mathrm{O} . h_{r}$ is hexagonal ice recovered from ice XI. $T_{0 d}^{\prime}$ is sufficiently close to $\frac{2}{7} T_{F d} \approx 79.14 \mathrm{~K}$ to suggest that the transition occurs when $\frac{9}{2} k_{B} T_{0 d}^{\prime}=h \nu_{1 r}$ supposing that $h \nu_{1 r}$ of ice $h_{r}$ may be slightly different from $h \nu_{1}$. It turns out that ice XI is $1-\mathrm{D}_{\chi}$.

For $\mathrm{H}_{2} \mathrm{O}, T_{0 d}^{\prime}-T_{0 h}^{\prime} \approx 4.8 \mathrm{~K}$ and $5 h \nu_{t h} / k_{B} \approx 4.83 \mathrm{~K}$ suggest $T_{0 h}^{\prime}=T_{0 d}^{\prime}-5 h \nu_{t h} / k_{B}$. Ice $h_{r h}$ is $6 \times 1-\mathrm{D}_{\chi}$ and the 6 -fold ground state cannot be realized at once through one and the same event in $1-\mathrm{D}_{\chi}$. When an induced state $h \nu_{1}$ of ice $\mathrm{XI}_{h}$ split at zero-energy cost into $\left|0_{+j}\right\rangle_{h} \pm\left|0_{-j}\right\rangle_{h}$ of ice $h_{r h}$ at $\Theta=0$, the condensate releases $-(6-1) h \nu_{t h}$ to complete the transition. (In the $x$-representation, the recovered ice is likely a shrunk empty hexagonal lattice with a ring structure similar to that of the intercalated rings of ice I $h$. Consequently, ice $h_{r}$ should be denser than ice I $h$.) Presumably, ice $h_{r}$ is more stable than ice $\mathrm{I} h$ in which the $7 \times 1-D_{\chi}$ character of water is imprinted. However, direct transitions between $6 \times 1-\mathrm{D}_{\chi}$ ice $h_{r}$ and $7 \times 1-\mathrm{D}_{\chi}$ ice $\mathrm{I} h$ or water, compatible with the least common dimensionality $42 \times 1-D_{\chi}$ and $h \nu_{1}$, is likely impossible under accessible $P, T$-conditions.

Upon cooling, the phase transition is exceedingly slow in the absence of a dopant. The transitions occur at $T=$ $T_{0}-\approx 14.5 \mathrm{~K}$ for both isotopomers and this shift could be simply related to the measurement protocol.

Water. - The quantum nature of water accounts for several "anomalous" properties.

(i) The stoichiometry of pure water accords with the mean hydration number of protons in $\mathrm{HCl}$-water solutions determined via freezing point depression: $\mathrm{H}^{+}\left(\mathrm{H}_{2} \mathrm{O}\right)_{6.7 \pm 0.7}$ 29 . The reported binding energy, $\approx(54 \pm 6) 10^{3} \mathrm{~J} . \mathrm{mol}^{-1}$ confirms the stability of this stoichiometry against thermal fluctuations.

(ii) In ice I $h$, tunnelling splitting distinctive of the inter- 
calated graph IV suggests shorter inter-vertexes distances shrinking 1/14 intercalated graph relative to 13/14 empty graph III. By contrast, the shrinking effect is a maximum in water. This is in line with the reduction of the molar volume of water relative to ice $\mathrm{I} h$. On the other hand, tunnelling in $6 \times 1-\mathrm{D}$ for recovered ice $h_{r h}$ suggests a shrunk empty hexagonal lattice denser than ice $\mathrm{I} h$.

(iii) The heat capacity of heavy water can be interpreted as a mixture of $\left(1-\gamma_{d}\right)$ separable and $\gamma_{d}$ nonseparable degrees of freedom. For example, the heat capacity of entangled pairs, i.e. $18 \mathcal{R}$, yields $\gamma_{d}=0.1$. For light water $\gamma_{h} \leq 0.01$ is insignificant in the range $T_{F}-T_{V} \cdot \gamma_{d}$ and $\gamma_{h}$ mirror different residence times in a well, that is $\tau=4 \nu_{1} \nu_{t}^{-2} \exp \left[h \nu_{1} /\left(k_{B} T\right)\right]\left[10\right.$. Since $\nu_{t d} / \nu_{t h} \ll 1$, marginal quantum correlations in $\mathrm{D}_{2} \mathrm{O}$ may be totally lacking in $\mathrm{H}_{2} \mathrm{O}$. However, the difference between $\mathrm{H}_{2} \mathrm{O}$ and $\mathrm{D}_{2} \mathrm{O}$ seems too small to compare with reported quantum effects in mixed isotope solutions 30,31 .

(iv) Below $T_{F}$, the heat capacity of supercooled $\mathrm{H}_{2} \mathrm{O}$ increases rapidly to a maximum value, $C_{\text {pmwh }} \approx 108$ J.mol ${ }^{-1} \cdot \mathrm{K}^{-1}(\approx 1.44 \times 9 \mathcal{R})$ just below $235 \mathrm{~K}$, that is about the homogeneous nucleation temperature $\left(T_{n h} \approx 228 \mathrm{~K}\right.$ at $\left.P_{a}\right)$. Below $T_{n h}$, Murphy and Koop 27 inferred from thermodynamic continuity between water and amorphous ice that $C_{p w h}$ should decrease until at something like $150 \mathrm{~K}$ it is near the heat capacity of ice $\mathrm{I} h$. The quantum scheme is straightforward. Firstly, $\gamma_{h}$ increases upon cooling in the range $T_{F}-T_{n h}$, until nonseparable degrees of freedom pervade the bulk. Secondly, below $T_{n h}$, entangled degrees of freedom merge into condensed sates and freezing out ensures convergence to $C_{p I h}$.

Vaporization. - This is regarded as a transition from the liquid $-A_{w}\left(T_{B}\right)=\mathcal{R} T_{B}\left[9-2 \ln \frac{3}{2}\right]-$ to the ground state of the steam condensate at $\mathcal{E}_{B}=9 \mathcal{R} T_{B}$ and $\Theta_{v}=0$ The exothermic enthalpy $-2 \mathcal{R} T_{B} \ln \frac{3}{2} \approx-2516 \mathrm{~J} . \mathrm{mol}^{-1}$ taken at face value would mean that the liquid should be unstable relative to the vapour condensate at any temperature, what is obviously not the case. The endothermic

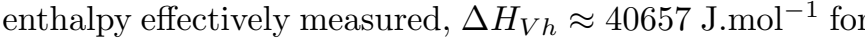

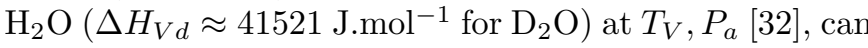
be attributed to a high level of degeneracy for the steam condensate. Every measurement-induced molecular-state is correlated with $\alpha_{v} \mathcal{N}_{\mathrm{A}}$ analogs, in such a way that the molar degeneracy is $\mathcal{N}_{\mathrm{A}}^{\alpha_{v}}$. Then, the unpredictability of realizations captured by Hartley-Shannon's entropy is $\mathbb{H}=\alpha_{v} \ln \mathcal{N}_{\mathrm{A}}$ and $\Delta H_{V}=T\left[\mathbb{H}-2 \mathcal{R} \ln \frac{3}{2}\right]$ is the energy cost to destroy the tetrahedral coordination graph of liquid water and to entangle the degrees of freedom into the steam condensate. $\ln \mathcal{N}_{\mathrm{A}}$ is a constant in J.mol ${ }^{-1}$ units, while $\alpha_{v}$ (fig. 3 is the nonlocal electronic correlation degree, in quantum words, or association degree, in chemical words, that is different in nature from the coordination degree.

At $298 \mathrm{~K}, \alpha_{v} \approx 2.75$ (from fig. 3) compares with $\alpha_{w} \approx$ $2.2 \pm 1.0$ 20. It can be inferred that the association degree representative of electronic correlations at a given $P, T$, is the same in both phases and can be referred to as " $\alpha$ ". The marginal isotope effect on $\alpha(\approx 2 \%)$ confirms that the association degree is not related to hydrogen bonding. The volume expansion of steam relative to water stems from the discontinuous jump of the coordination degree only.

As $T \longrightarrow T_{F}, \alpha$ increases. It can be extrapolated that $\alpha \approx 4$ for gas-phase clusters realized at very low temperature in jet expansions, as well as for ice $\mathrm{I} h$ at $5 \mathrm{~K}$. This is corroborated by identical tunnelling splittings in both phases 4]. As $T \longrightarrow T_{c}, \alpha$ decreases and vanishes abruptly at $T_{c}$. In fact, the liquid phase is unstable for $\alpha<\alpha_{c}=2 \mathcal{R} \ln \frac{3}{2} / \ln \mathcal{N}_{\mathrm{A}} \approx 0.12$. The red-dashed curve in fig. 3 suggests cooperative effects through thermal population of $h \nu_{1}$ in the range $300-600 \mathrm{~K}$.

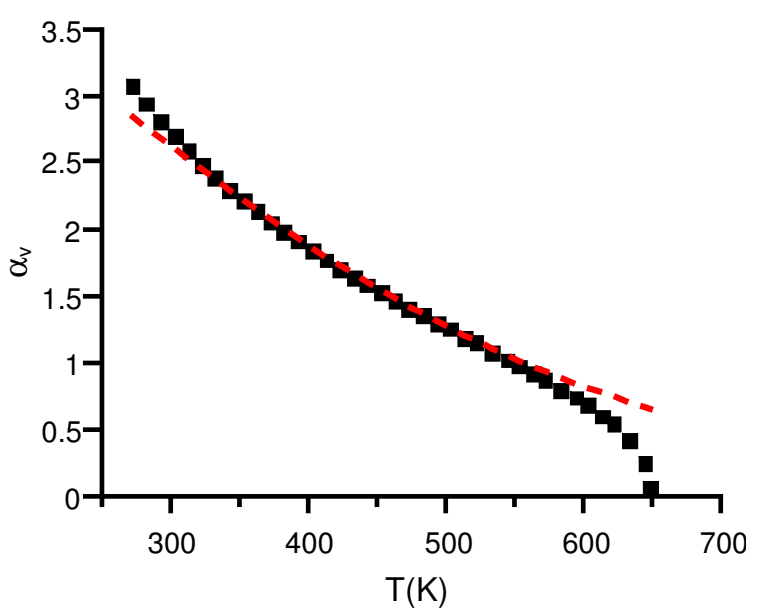

Fig. 3: The association degree deduced from the measured enthalpy of vaporization 33. $\mathbf{\square}: \alpha_{v}(T)=\left(\Delta H_{V}(T) / T+\right.$ $\left.2 \mathcal{R} \ln \frac{3}{2}\right) / \ln \mathcal{N}_{\mathrm{A}} . \quad \mathcal{N}_{\mathrm{A}} \approx 6.022 \times 10^{23} . \quad$ Red-dashed: $\alpha=$ $4\left[1-\exp \left(-h \nu_{1} / k_{B} T\right)\right] * *\left[1+\left(T-T_{F}\right) /\left(T_{c}-T_{F}\right)\right]$.

Sublimation. - At the melting point, the enthalpy

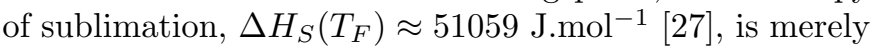
$\Delta H_{F}\left(T_{F}\right)+\Delta H_{V}\left(T_{F}\right) \approx 51058$ J. A single-step transition between ice and steam is prohibited, as the ground-state of steam, $\mathcal{E}_{F}=9 \mathcal{R} T_{F}$, is twice the eigenenergy of ice. The liquid phase and hence the intermediate phase of fusion are necessary steps in the sublimation process.

Conclusion. - Heat capacities, latent heats, neutron scattering, transistion temperatures and dielectric measurements converge consistently to the conclusion that ices and steam at thermal equilibrium are bulk-scale condensates with continuous spacetime-translation symmetry, whereas water in the range $T_{B}-T_{F}$ is a quantum liquid with broken time-translation symmetry. It is shown that the properties of the phases are archetypal of a quantum material, instead of anomalies of a classical model. As there is no evidence to suggest any upper bound for the quantum theory, relative to the temperature, or the physical state, the framework should be confronted with the 
whole $P, T$-phase-diagram of water and other materials eventually.

The quantum framework is self-contained. It is a sound alternative to the unsolvable many-body problems inherent to microrealism. Nonlocality paves the way to a new vista of physics, chemistry and molecular biology in water.$$
* * *
$$

I am grateful to an anonymous referee for valuable comments.

\section{REFERENCES}

[1] Chaplin M., http://www1.lsbu.ac.uk/water/ hexagonal_ice.html, (2017).

[2] Bove L. E., Klotz S., Parciaroni A. and Sacchetti F., Phys. Rev. Letters, 103 (2009) 165901.

[3] Keutsch F. N. and Saykally R. J., PNAS, 98 (2001) 10533.

[4] See supplementary material for tunnelling in clusters.

[5] Ball P., Nature, 452 (2008) 291.

[6] Giese T. J. and York D. M., J. Phys.: Condens. Matter 29 (2017) 383002 (14pp), 29 (2017) 383002 (14pp).

[7] Mukhopadhyay A., Cole W. T. and Saykally R. J., Chem. Phys. Letters, 633 (2016) 13.

[8] Drechsel-Grau C. and Marx D., Phys. Rev. Letters, 112 (2014) 148302.

[9] Leggett A. J., J. Phys.: Condens. Matter, 14 (2002) R415.

[10] Fillaux F. and Cousson A., Eur. Phys. J. B, 89 (2016) 72.

[11] Fillaux F. and Cousson A., Chem. Phys., 479 (2016) 26.

[12] Plotnitsky A., Phys. Scr.T, 163 (2014) 01402 (20pp).

[13] Feistel R. and Wagner W., J. Phys. Chem. Ref. Data, 35 (2006) 1021.

[14] Bernal J. D. and Fowler R. H., J. Chem. Phys., 1 (1933) 515.

[15] Pauling L., JACS, 57 (1935) 2680.

[16] Giauque W. F. and Stout J. W., JACS, 58 (1936) 1144.

[17] Kuhs W. F. and Lehmann M. S., J. Phys. Chem., 87 (1983) 4312.

[18] Lishchuk S. V., Malomuzh N. P. and Makhlaichuk P. V., Phys. Letters A, 375 (2011) 2656.

[19] Bol W., J. Appl. Cryst., 1 (1968) 234.

[20] Wernet P., Nordlund D., Bergmann U., Cavalleri M., Odelius M., Ogasawara H., Näslund L. ., Hirsch T. K., Ojamäe L., Glatzel P., Pettersson L. G. M. and Nilsson A., Science, 304 (2004) 995.

[21] Smirnova N., Bykova T., Durme K. V. and Mele B. V., J. Chem. Thermodynamics, 38 (2006) 879.

[22] Verma M. P., Computers Geosci., 29 (2003) 1155.

[23] Senesi R., Romanelli G., Adams M. and Andreani C., Chem. Phys., 427 (2013) 111.

[24] Li J., J. Chem. Phys., 105 (1996) 6733.

[25] Fillaux F., Tomkinson J. and Penfold J., Chem. Phys., 124 (1988) 425.

[26] Soper A. K., Chem. Phys., 258 (2000) 121.
[27] Murphy D. M. and Koop T., Q. J. R. Meteorol. Soc., 512 131 (2005) 1539.

[28] Yen F. and Chi Z., PCCP, 17 (2015) 12458.

[29] Zavitsas A. A., J. Phys. Chem. B, 105 (2001) 7805.

[30] WeingÄrtner H. and Chatzidimitriou-Dreismann C. A., Nature, 346 (1990) 548.

[31] Chatzidimitriou-Dreismann C. A., Krieger U. K., Moiler A. and Stern M., Phys. Rev. Letters, 75 (1995) 3008.

[32] Marsh K. N., (Editor) Recommended Reference Materials for the Realization of Physicochemical Properties (Blackwell, Oxford) 1987.

[33] Lange's Handbook of Chemistry (McGraw-Hill Professional) 1998 p. 1476. 